\title{
Proyectos de aula en la gestión del conocimiento en la Universidad Nacional Hermilio Valdizán, Huánuco, Perú
}

\author{
Projects of classroom in the knowledge management in the national university \\ Hermilio Valdizán, Huánuco, Peru \\ Nicéforo Bustamante P. ${ }^{1}$, Raúl Aliaga C. \\ Universidad Nacional Hermilio Valdizán de Huánuco, Perú
}

(RECIBIDO 10 /09/2016, AcEPTADo 29 /10/2016)

\begin{abstract}
RESUMEN
Los rasgos distintivos de la didáctica universitaria valdizana son la transmisión de información con escasa gestión del conocimiento por parte del alumno y del docente. El objetivo fue renovar la didáctica a través de proyectos de aula en la gestión del conocimiento. La metodología consistió en la práctica de la socio formación, centrado en el trabajo personalizado, investigación acción y el trabajo colaborativo. Los resultados son la valoración de la metacognición, la importancia del talento individual, y la socialización del conocimiento en las brigadas de aprendizaje. Se tiene por conclusión que, el proyecto de aula en clase es una estrategia didáctica que permite discernir niveles de aprendizaje, desde el trabajo individual, aprendizajes en brigadas y socialización del conocimiento.
\end{abstract}

Palabras clave: Gestión, conocimiento, proyecto, brigadas, socioformación

\begin{abstract}
The distinctive features of valencian university didactics are the transmission of information with little knowledge management by the student and the teacher. The objective was to renew the didactics through classroom projects in the management of knowledge. The methodology consisted of the practice of the training partner, focused on personalized work, research action and collaborative work. The results are the assessment of metacognition, the importance of individual talent, and the socialization of knowledge in learning brigades. We conclude that the classroom project in the classroom is a didactic strategy that allows for discerning levels of learning, from individual work, learning in brigades and socialization of knowledge.
\end{abstract}

Keyword: Management, knowledge, project, brigades, socio-formation 


\section{INTRODUCCIÓN}

En las cátedras universitarias sigue primando las clases magistrales expositivas, repitiendo información ya existente en los textos universitarios, revistas, artículos, antologías, y los otros repositorios como el internet a través de las páginas web y los blog. Esta metodología se ha hecho rutina y se caracteriza por actividades como la exposición de clases con temas seleccionados y consultas a diversos autores, asignación de trabajos a los estudiantes para que realicen monografías, consultas bibliográficas y exposiciones en fechas programadas, elaboración de organizadores, y lecturas individualizadas. Estas estrategias didácticas perduran hasta nuestros días en la mayoría de las universidades peruanas y en particular en la Universidad Nacional Hermilio Valdizán (UNHEVAL). La mayoría de los futuros profesionales se dedican a repetir información proporcionado por sus docentes, muchas veces desfasados en su contenido y vigencia, descuidando el descubrir o desarrollar talento humano, con escasa contribución de nuevos conocimientos. Se practica una didáctica inservible para los requerimientos del mercado ocupacional y para el desempeño de los futuros profesionales. En el caso de Educación se viene repitiendo los modelos establecidos por el Banco Mundial a través de Ministerio de Educación. Esta realidad nos indujo a realizar un trabajo basado en proyectos. Para el efecto nos propusimos como objetivo experimentar el Proyecto de Aula en la Gestión del Conocimiento, el mismo que lo desarrollamos entre los años de 2014, 2015 y actualmente se continúa. Como es evidente los proyectos de aprendizaje no son nada nuevos, Kilpatrick allá por 1918 en los Estados Unidos, para el desarrollo de aprendizajes a través de proyectos productivos, con énfasis en el interés personal del aprendiz, se obtuvo resultados sustantivos para afianzar experiencias pedagógicas. En el nivel universitario se tiene escasa referencia, uso y resultado, salvo lo que se viene experimentando en el modelo de socioformación en México, que afirma:

"La primera meta de la socio-formación es que las personas posean un sólido proyecto ético de vida. Esto es, que tengan un propósito claro en sus vidas, busquen la realización personal, trabajen con laboriosidad y perseverancia en el logro de las metas y actúen con base en los valores universales" (Tobón, 2013, p.10).

$\mathrm{Al}$ revisar los manuales de didáctica sugeridos por los docentes universitarios, hallamos escasas experiencias de proyectos utilizados en aula para la gestión de nuevos conocimientos. La mayoría de los textos o propuestas inducen a los estudiantes a memorizar y solo repetir la información de tipo logocéntrico. Diez-Canseco (1984) afirma: "el profesor logocéntrico está centrado en la ciencia y, aunque no sea investigador, sino es un mero transmisor del saber ya constituido"(p. 84). De lo dicho se constata que la práctica docente valdizano, aún se halla vigente en contenido y forma. En este contexto en los foros académicos nacionales e internacionales de los últimos 20 años se empezó, a discutir y plantear las políticas educativas de parte del Estado, políticas institucionales universitarias, desarrollar currículo por competencias, capacidades, dominios de aprendizaje en las aulas universitarias, manejo de la Tecnología de la Información y Comunicación, (TIC), la Tecnología de Aprendizaje y Conocimiento (TAC). Tecnología de Emprendimiento y 
la Participación (TEP). Algunos recomendaban hacer una reingeniería del trabajo universitario para obtener la calidad profesional, por su parte las instituciones tutelares como la ex Asamblea Nacional de Rectores (ANR), el Sistema Nacional de Evaluación Acreditación y Certificación de la Calidad Educativa (SINEACE), a través de la Ley 28740, el ex Consejo de Evaluación, Acreditación y Certificación de la Calidad de la Educación (CNEAU), así como el fenecido Consejo Regional Interuniversitario del Centro (CRI) sugerían hacer una nueva filosofía del trabajo universitario basado en los principios del Aseguramiento de la Calidad. Todas estas premisas nos permitieron la innovación didáctica en nuestra práctica docente a través de proyectos de aula en la gestión de nuevos conocimientos, (ser, hacer, aprender y emprender) siendo el principal protagonista el estudiante, lo que les permite desarrollar competencias personales profesionales y generar saberes a través de la metacognición, (acción evocación y reflexión) mediante el trabajo colaborativo en las brigadas de aprendizaje, la autorrealización a través de la gestión de sus propios conocimientos. Realidad que nos condujo a reflexionar y reformular nuestra didáctica universitaria, a la que denominamos: La Investigación Formativa en estudiantes de la Especialidad de Ciencias Histórico Sociales y Geográficas (Proyectos de aula como Gestión del Conocimiento). Iniciamos el trabajo esporádicamente desde el 2009, con los alumnos de la promoción de cada año, lo que nos dio resultados $\mathrm{Y}$ experiencias sustantivos y que a partir del II semestre del año lectivo 2014 iniciamos de manera planificada como efecto de una evaluación curricular, cuyos preliminares enunciamos:

\section{MÉTODO}

Se utilizó la técnica del fichaje con almacenamiento electrónico: análisis documental en relación al marco conceptual de gestión del conocimiento con proyectos de aula en concordancia a la investigación hermenéutica. Como material de trabajo se utilizó los celulares de uso personal para grabar, filmar, retomar el uso de fichas impresas y cuaderno de notas que nos sirvió para coadyuvar la técnica del fichaje, en el recojo de información dentro del trabajo de campo. El análisis sirvió para buscar y seleccionar documentos que refuercen nuestro referente teórico. Estas dos técnicas sirvieron para el análisis de los resultados.

En el proceso de la investigación y en el informe se tomó como modelo la metodología de la cartografía conceptual propuesta por Tobón $(2004,2015 a)$, que se complementó con la hermenéutica. Ambas metodologías, permitieron seguir un conjunto de procesos, desde la observación preliminar hasta elaborar un marco conceptual definido.

\section{PROCEDIMIENTOS DEL TRABAJO}

Para ejecutar el proyecto, primero se organizó a los estudiantes en brigadas de trabajo, a fin de cumplir actividades en los centros de información como complejos 
arqueológicos, archivos, museos, bibliotecas, hemerotecas, navegar en las páginas de la web, usar bibliotecas virtuales a través de enlaces, visitar comunidades, entrevistarse con autoridades y personalidades, tomar fotografías, elaborar mapas, croquis, esquemas, manejar herramientas como las Tic, Tac y el Tep; hacer uso de las redes sociales, hasta llegar a elaborar informes, artículos, monografías, tesinas e intercambiar sus saberes con sus pares. Requisito primordial uso de medios y herramientas personales

Basándose en estos procedimientos se organizó nuestra cartografía conceptual que indicamos a continuación.

\section{CUADRO N. ${ }^{\circ} 1$}

CARTOGRAFÍA CONCEPTUAL PARA ESTRATEGIAS DE TRABAJO

\begin{tabular}{|c|c|c|c|}
\hline $\begin{array}{l}\text { Unidad de } \\
\text { análisis }\end{array}$ & Descripción & Componentes & Logro \\
\hline Concepto & $\begin{array}{l}\text { ¿En qué consiste el pro- } \\
\text { yecto de aula en la ges- } \\
\text { tión del conocimiento? }\end{array}$ & $\begin{array}{l}\text { Conceptos sobre la impor- } \\
\text { tancia de los proyectos de } \\
\text { aula en la gestión del co- } \\
\text { nocimiento y su proceso } \\
\text { histórico. }\end{array}$ & $\begin{array}{l}\text { Fichas. } \\
\text { Antecedentes. } \\
\text { Importancia. }\end{array}$ \\
\hline $\begin{array}{l}\text { Organización } \\
\text { de brigadas }\end{array}$ & $\begin{array}{l}\text { ¿Cuáles son los rasgos } \\
\text { que caracterizan a las } \\
\text { brigadas de estudio en } \\
\text { la gestión del conoci- } \\
\text { miento? }\end{array}$ & $\begin{array}{l}\text {-Equipo interdisciplinario } \\
\text { orientado a la búsqueda de } \\
\text { la información. }\end{array}$ & $\begin{array}{l}\text { Organización. } \\
\text { Liderazgo- } \\
\text { Desarrollo de ac- } \\
\text { titudes. }\end{array}$ \\
\hline $\begin{array}{l}\text { Aspecto moti- } \\
\text { vacional }\end{array}$ & $\begin{array}{l}\text { ¿Cómo entienden la } \\
\text { importancia de los pro- } \\
\text { yectos en la gestión del } \\
\text { conocimiento? }\end{array}$ & $\begin{array}{l}\text { Valor y trascendencia de } \\
\text { los proyectos en la forma- } \\
\text { ción de la vida. }\end{array}$ & $\begin{array}{l}\text { Proyecto de vida } \\
\text { personal y profe- } \\
\text { sional. } \\
\text { Actitudes voliti- } \\
\text { vas y conativas. }\end{array}$ \\
\hline $\begin{array}{l}\text { Contribución } \\
\text { individual }\end{array}$ & $\begin{array}{l}\text { ¿Cuáles son las contri- } \\
\text { buciones individuales en } \\
\text { el tema de estudio? }\end{array}$ & $\begin{array}{l}\text { Aportes y contribuciones } \\
\text { del talento individual so- } \\
\text { bre el tema elegido. }\end{array}$ & Propuestas \\
\hline $\begin{array}{l}\text { Discusión en } \\
\text { el seno de la } \\
\text { brigada }\end{array}$ & $\begin{array}{l}\text { ¿Cómo contribuyen a su } \\
\text { brigada cada uno de los } \\
\text { integrantes del tema de } \\
\text { estudio? }\end{array}$ & $\begin{array}{l}\text {-Contribución individual y } \\
\text { colectiva sobre el tema en } \\
\text { estudio. }\end{array}$ & $\begin{array}{l}\text { Alternativas de } \\
\text { solución. }\end{array}$ \\
\hline $\begin{array}{l}\text { Elaboración } \\
\text { del informe } \\
\text { académico }\end{array}$ & $\begin{array}{l}\text { ¿En qué consiste el in- } \\
\text { forme académica de la } \\
\text { brigada? }\end{array}$ & $\begin{array}{l}\text {-Se elabora el primer bo- } \\
\text { rrador del informe }\end{array}$ & $\begin{array}{lr}\text { Descripción } & y \\
\text { contrastación } & \text { del } \\
\text { nuevo saber. } & \\
\end{array}$ \\
\hline $\begin{array}{l}\text { Sustentación } \\
\text { en plenaria }\end{array}$ & $\begin{array}{l}\text { ¿De qué manera se da la } \\
\text { presentación de trabajos } \\
\text { en la plenaria? }\end{array}$ & $\begin{array}{l}\text {-Cada jefe de brigada ex- } \\
\text { pone al pleno y se produce } \\
\text { el debate entre las briga- } \\
\text { das, así como recibe las } \\
\text { sugerencias de los miem- } \\
\text { bros de la plenaria y del } \\
\text { docente. }\end{array}$ & $\begin{array}{l}\text { Fundamentación } \\
\text { Argumentación, } \\
\text { defensa de ideas y } \\
\text { propuestas. }\end{array}$ \\
\hline
\end{tabular}




\begin{tabular}{|c|c|c|c|}
\hline Metodología & $\begin{array}{l}\text { ¿Cuáles son los ras- } \\
\text { gos metodológicos que } \\
\text { abordan los proyectos } \\
\text { de aula en la gestión del } \\
\text { conocimiento? }\end{array}$ & $\begin{array}{l}\text { Se describe de manera } \\
\text { pormenorizada los proce- } \\
\text { dimientos a seguir. }\end{array}$ & $\begin{array}{l}\text { M a p e o } \\
\text { metodológico }\end{array}$ \\
\hline Conclusión & $\begin{array}{l}\text { ¿Cuál son los aportes de } \\
\text { trascendencia del tema } \\
\text { de estudio que le sea } \\
\text { significativo en su vida } \\
\text { profesional? }\end{array}$ & $\begin{array}{l}\text { Precisar con ejemplos los } \\
\text { aportes de los brigadistas } \\
\text { y de los integrantes de la } \\
\text { plenaria en la gestión del } \\
\text { nuevo conocimiento.. }\end{array}$ & $\begin{array}{l}\text { Aportes significa- } \\
\text { tivos: innovadores } \\
\text { y emprendedores. }\end{array}$ \\
\hline $\begin{array}{l}\text { Publicación } \\
\text { de resultados }\end{array}$ & $\begin{array}{l}\text { ¿De qué manera socia- } \\
\text { lizan el nuevo conoci- } \\
\text { miento? }\end{array}$ & $\begin{array}{l}\text { Publican en los medios } \\
\text { informativos como bole- } \\
\text { tines, periódico mural, } \\
\text { informativo electrónico, } \\
\text { revistas estudiantiles, mo- } \\
\text { nografías etc. }\end{array}$ & $\begin{array}{l}\text { Presentación de } \\
\text { resultados y li- } \\
\text { bros. }\end{array}$ \\
\hline
\end{tabular}

\section{Procedimientos seguidos en la investigación}

Elaboración y fundamentación de los proyectos, organización de brigadas de estudio, orientaciones metodológicas a los estudiantes en el aula el primer día de clases, propuesta de temas de estudio por los alumnos, recojo individual de información, discusión e intercambio de experiencias en las brigadas, elaboración del trabajo preliminar, sustentación e interaprendizaje de experiencias en plenaria, conclusiones del pleno y del docente responsable de la cátedra, y publicación de resultados.

\section{RESULTADOS}

\section{Evaluación curricular}

En el primer semestre del 2014 se hizo la evaluación curricular como parte de nuestra actividad docente universitaria, se convocó a la plana docente, representantes de estudiantes, grupos de interés, egresados y personal administrativo. La evaluación simultánea arribo a conclusiones precisas, siendo la de mayor déficit el aspecto metodológico de los docentes. En el Informe Curricular de la Facultad de Ciencias de la Educación de la Universidad Nacional Hermilio Valdizán (UNHEVAL, 2014), dice: "la metodología está centrada en el modelo expositivo y la transmisión de contenidos y no se desarrolla las competencias exigidas en el currículo de formación profesional" (p. 13). Teniendo como referente dicho informe, reformulamos nuestra metodología de trabajo a través de la investigación formativa en su modalidad proyectos de aula como gestión del conocimiento; para ello se seleccionó como muestra representativa los cursos: Perú Republicano en el Contexto Mundial S. XIX y XX e Historia del Perú en el Contexto de Liberación Mundial S. XVII y XVIII. Estos cursos corresponden al 5to y 4to año, respectivamente. Para el trabajo los alumnos fueron organizados en brigadas de aprendizaje de 3 a 5 personas. Estas brigadas se constituyeron en gestoras de su propio apren- 
dizaje y conocimiento, para ello elaboraron su micro proyecto de aprendizaje en aula con temas que ellos consideraban de interés para su formación profesional, para este fin fue indispensable la presentación del sílabo del docente, que previamente fue elaborado en jornada conjunta con los alumnos, para luego ser validado por las autoridades académicas en función a las exigencias de las competencias propuestas en el currículo de formación profesional. Los estudiantes escogieron los temas de manera libre y autónoma; el docente cumplió la labor de monitor y orientador y evaluador del procedimiento a seguir para la eficacia y eficiencia del trabajo estudiantil.

\section{Rol del docente}

Para desarrollar los proyectos de aula en la gestión del conocimiento, el docente, como parte estratégica, debe tener una nueva forma de actuar en el escenario de su didáctica y estar a la altura de un gestor de conocimiento:

Que los profesores universitarios son distintos a otros profesores y que enseñar en la universidad es distinto a enseñar en otros contextos educativos, y que los parámetros que se pueden analizar para medir la enseñanza en otros contextos no sirven para la enseñanza universitaria, porque el propio contexto, la naturaleza del conocimiento a desarrollar, los propósitos formativos, están allí asentado el edificio de toda la formación en la universidad (Zabalza, 2005, p.24).

Tomando en cuenta los grupos etarios, las experiencias y procedencia, (en el actuar andrológico) en este contexto el docente opta por una nueva forma de ver el aprendizaje, internaliza en el aula con sus estudiantes algunos documentos básicos, entre ellos: primero el currículo de formación profesional, donde están definidos los perfiles de competencia del egresado; segundo el sílabo, en el que están seleccionados las competencias, capacidades y dominios a lograrse; asimismo los marcos normativos inherentes a la función estudiantil, donde están precisados los deberes y responsabilidades del estudiante; y tercero, el horario de clases en bloque que tiene como finalidad el uso óptimo del tiempo, complementario a esto se les indica los principales centros de información. Un hecho importante es llegar al consenso en esta fase; la experiencia nos dice que no se debe imponer esta metodología sino sensibilizar el sentido conativo; es necesario fijar conjuntamente con los estudiantes el código de responsabilidades, donde todos deben participar a fin de evitar posteriores desacuerdos. Igualmente el docente expone con bastante claridad a sus estudiantes sobre los rasgos de los logros (artículos, monografías, informes, composiciones, antologías, tesinas, módulos, etc.) es de primordial importancia que las brigadas tengan claridad de los rasgos de cada uno de los documentos referidos, pues el aprendizaje y la construcción de sus nuevos conocimientos lo plasmaran en los documentos aludidos. Finalmente estos documentos después de ser informados e internalizados, forman parte del portafolio universitario del estudiante y del docente. 


\section{Proyectos de aula}

El proyecto de aula es una estrategia didáctica que permite a los estudiantes a gestionar sus aprendizajes, conducentes a la gestión de un nuevo conocimiento (proponer, conocer, actuar y reflexionar). Sobre el particular ya los estudiosos como (Biggs) 2005 nos dice: "La enseñanza es individual, de ahí la necesidad que tenemos de ajustar nuestras decisiones docentes a nuestra materia, a los recursos disponibles, a nuestros estudiantes y a nuestras propias virtudes y defectos como profesores" (pp. 19-28). Concordando con ello, renovamos nuestra estrategia que orienta al logro de competencias a través del desarrollo de habilidades, afectivas, conductuales, Y sociales, sobre todo privilegiar la práctica de las habilidades de pensamiento consistentes en el manejo de la conceptualización, comprensión, generalización, clasificación, secuenciación, comparación y contrastación, de análisis, síntesis, abstracción, resolución de problemas, toma de decisiones y sobre todo el manejo de las fuentes de información, la práctica del emprendimiento y la innovación. El proyecto permite al estudiante actuar y tomar decisiones sobre su propio aprendizaje, es decir realiza una microinvestigación que le permite manejar información pero sobre todo gestionar su nuevo conocimiento.

\section{Estrategias de trabajo y aprendizaje}

El proyecto de aprendizaje en el aula, conducente a la gestión de un nuevo conocimiento, como sostiene Tobón (2013), propone la socioformación: formar y consolidar el proyecto ético de vida, tener emprendimiento, desarrollar las competencias necesarias para afrontar los retos del contexto y trabajar de manera colaborativa es una estrategia que coadyuva a la investigación formativa, donde el estudiante debe cumplir un conjunto de acciones. "La enseñanza 'funciona' haciendo que los estudiantes se comprometan en actividades relacionadas con el aprendizaje y que les ayuden a alcanzar los objetivos concretos establecidos para la unidad o asignatura, como teorizar, crear nuevas ideas, reflexionar, aplicar, resolver problemas, memorizar" (Biggs, 2005, pp.19-28). Pues para ejecutar su proyecto el alumno debe realizar una serie de actividades en diversos centros y repositorios de información. Por su parte Benites (2010) afirma:

Las Tecnologías de la Información y la Comunicación (TIC) juegan un papel fundamental en este nuevo espacio, como herramientas que pueden facilitar tanto la implementación de nuevas metodologías como el aprendizaje del alumno y la comunicación interpersonal alumno-alumno y alumno-profesor (p. 2).

Es decir se potencia a través de estos medios el aprendizaje colaborativo en las brigadas estudiantiles y la gestión de sus propios conocimientos, y estos recursos que les permite a los brigadistas el manejo de herramientas y otras técnicas investigativas, dependiendo de la naturaleza del tema en estudio.

Los estudiantes, teniendo localizado su centro de información y haciendo uso de las técnicas e instrumentos de trabajo recogen los datos, a lo que denominamos el aprendizaje preliminar, donde el alumno de manera personal experimenta con la 
realidad y percibe de cerca el enorme corpus informativo. Estas evidencias los registra usando herramientas convencionales o electrónicas y se constituye el primer aprendizaje personal; esta información pasa a una segunda fase de trabajo, cuando el aprendizaje individual es socializado en su brigada de trabajo, con quienes, analiza y discute el hallazgo. Esta segunda fase es conocida como el aprendizaje de la brigada y en ella interviene el docente con algunas aclaraciones, si el caso amerita, de lo contrario el docente se reserva hasta el final. Un rasgo esencial de los proyectos de aula es respetar la autonomía e independencia de la brigada sobre todo en los pareceres y conclusiones. Concluido el trabajo la brigada presenta un informe al plenario con la finalidad de construir el nuevo conocimiento, donde los alumnos participan con sus preguntas, observaciones; siendo el rol fundamental de los miembros de la plenaria el análisis, la socialización y el juicio crítico de sus integrantes; en esta fase la intervención del docente es enfático con ampliación, aportes y recomendaciones sobre todo en la parte formal de los informes de aprendizaje que se presentan.

\section{La investigación formativa como estrategia de aprendizaje}

Además de ser una estrategia de aprendizaje, en la gestión del conocimiento, los proyectos de aula se constituyeron en generadores de lo que se llama la investigación formativa. Restrepo (s.f) afirma:

Otra forma de trabajar la investigación formativa es el trabajo de los estudiantes con el profesor que investiga, aprendiendo a formular problemas y proyectos, a idear hipótesis, a diseñar su metodología, a saber recopilar información, a procesar datos, a discutir, argumentar, interpretar, inferir y defender resultados (p.11).

Efectivamente, en los proyectos de aula, las brigadas, dentro de su proceso de formación profesional, adquieren hábitos de investigador. Las brigadas están organizados alrededor de un comité directivo que promueve actitudes, valores, comparte e intercambia experiencias en el manejo de métodos, técnicas en torno al objeto de estudio; en este caso relacionados a la Ciencias Histórico Sociales y Geográficas.

En un artículo de la Universidad Politécnica de Madrid, (2008) se afirma:

Con este método los alumnos tienen que trabajar en equipo desde el principio y desarrollar un proyecto que solucione la situación presentada planificando la actuación, distribuyendo tareas, investigando, analizando los contextos involucrados, desarrollando el plan establecido, evaluando las posibles consecuencias, previendo los éxitos (p. 5).

Asumiendo actitudes de curiosidad, búsqueda permanente de la lectura y la conversación a cerca de los nuevos hallazgos y novedades científicas, el planteamiento de problemas, formulación de hipótesis, el trabajo intelectual en brigadas entre otras actitudes investigativas. 


\section{Práctica de valores}

El proyecto induce a asumir actitudes de trabajo colaborativo generando la autocrítica, crítica, debate, código de ética, los protocolos investigativos, la autoevaluación, el intercambio de pareceres, el respeto por las ideologías y por las creencias de los compañeros de aula. Marquesán (2008) dice:

Especial interés merece la igualdad que debe darse entre hombres y mujeres, analizando las causas y los factores responsables de la discriminación de estas últimas, su valoración desde los principios de la dignidad de la persona y la igualdad en libertad (p.15).

En la brigadas los participantes actúan en igualdad de condiciones académicas, de valoración de género, raza, color, idioma y credo bajo el trato equitativo del docente con sus estudiantes, y, entre los alumnos, el respeto de los unos por los otros, y sobre todo la valoración de su cultura, su espacio y su identidad conducente a su formación personal profesional con respeto y dignidad hacia los demás..

\section{Manejo de métodos y técnicas}

El proyecto de clases en aula induce al alumno a una nueva forma de aprendizaje. "Una idea clara es la idea del aprendizaje autónomo de los estudiantes, es decir la didáctica tiene que generar espacios, instrumentos, estrategias, etc., que tengan mucho que ver con lo que es este trabajo autónomo de los estudiantes" (Zabalza, 2005 , p. 24). Efectivamente, la didáctica tradicional del uso exclusivo de métodos y técnicas, ha dado paso a una nueva forma de hacer el conocimiento, empezando por la prospección y la observación de los hechos y fenómenos, por lo general nuestros alumnos se formulan hipótesis sobre su tema o problema de estudio, luego van en pos de la verificación y la experimentación, para luego contrastar y abstraer la información, hasta llegar a la generalización de los conceptos precisos y claros sobre su tema de estudio.

\section{Inicios de la investigación}

Nuestra experiencia con los estudiantes del 4to y 5to año de la Especialidad de Ciencias Histórico Sociales y Geográficas ha evidenciado que la investigación juega un papel de primer orden en el aprendizaje y la construcción del conocimiento. Evidentemente lo entendemos como parte de la ciencia agógica en su nivel pedagógico, hebegógico hasta andragógico. En este contexto los estudiantes empiezan por la investigación exploratoria. Gestionar el conocimiento en el aula de clase requiere contar con datos, y la perseverancia tanto del docente como de los estudiantes. Indudablemente es un avance significativo en el desarrollo de determinadas competencias de formación profesional, la innovación, investigación, el emprendimiento, la crítica y la autocrítica, pero sobre la generación de un nuevo saber, es decir el estudiante no solo debe quedarse en la recepción de la información sino debe generar nuevos saberes con la información obtenida, tal como exige la sociedad del conocimiento. Todo esto adagio y experiencia permitirá al futuro 
profesional en educación innovar sesiones de aprendizaje para dinamizar y gestionar nuevos conocimientos en sus futuros alumnos.

\section{Innovando la didáctica y la investigación}

Uno de los grandes problemas con los estudiantes de la muestra fue la asimilación de la información que desarrollaba en las aulas, casi durante toda nuestra experiencia docente, se preparaba las clases de manera sistemática, consultando diversos autores, para luego disertar un tema en el aula de clase; Los estudiantes, ocasionalmente hacían preguntas, las mismas que se les respondía hasta complacer sus inquietudes, pero después de dos o tres semanas ellos se habían olvidado; solo para las evaluaciones programadas repasaban sus apuntes, y muchos de ellos acertaban con las respuestas y obtenían notas aprobatorias. Es decir, la cátedra estaba centrada en la transferencia de contenidos que previamente como docente había asimilado; con este tipo de cátedra no se lograba desarrollar ninguna competencia, ni habilidades cognitivas, afectivas, sociales ni culturales; al cambiar la estrategia docente por los proyectos de clase en aula cambió todo. Es decir la didáctica pasó de ser una mera transmisora a una didáctica de tipo operativa por parte de los estudiantes, porque empezaron a ser partícipes de su propio aprendizaje y sobre todo comenzaron a desarrollar competencias como el trabajo en brigadas, investigando en los repositorios de la información, manejo y uso de las técnicas e instrumentos de investigación, procesamiento de datos, manejo de métodos como la heurística y la hermenéutica, intercambio de información, y la práctica del saber compartido; logrando concordando con lo que postula el Ministerio de Educación (2015) para educación Básica, que sostiene: "El estudiante, a partir de un problema histórico, elabora explicaciones con argumentos basados en evidencias. En estas emplea adecuadamente conceptos históricos. Explica y jerarquiza las causas de los procesos históricos relacionándolas con las intencionalidades de los protagonistas" (p.15). Evidentemente este nuevo procedimiento del aprendizaje permite a los estudiantes a través de las brigadas generar sus propios conocimientos y aprendizajes, centrándose la didáctica en el conjunto de actividades de los estudiantes y al mismo tiempo iniciándose el proceso de la investigación.

\section{Experiencias de campo}

Durante nuestro trabajo con las brigadas estudiantiles logramos algunos datos preliminares que se expresan en los resultados finales de sus aprendizajes y la satisfacción de su permanencia en su etapa de estudios. En la tabla y gráfico $\mathrm{N}^{\circ} 1$, respectivamente, se observa que los estudiantes en su mayoría terminaron su carrera exactamente en cinco años a diferencia de períodos lectivos anteriores, cuando se tenía una alta deserción. Igualmente sus aprendizajes muestran calificaciones buenas y muy buenas. 
Tabla 1. Situación académica de los alumnos del 4to y 5to años en los cursos de Perú republicano y liberación mundial s. XVII y XVIII

\begin{tabular}{lcc}
\hline \multicolumn{1}{c}{ CONDICIÓN } & 4to & 5to \\
\hline Matriculados & 18 & 8 \\
\hline Aprobados & 16 & 6 \\
\hline Retirados & 2 & 2 \\
\hline Desaprobados & 0 & 0 \\
\hline
\end{tabular}

Fuente: Unidad de Procesos Académicos de la UNHEVAL, 2015.

Figura 1. Situación académica de los alumnos del 4to y 5 to años en los cursos de Perú republicano y liberación mundial s. XVII y XVIII

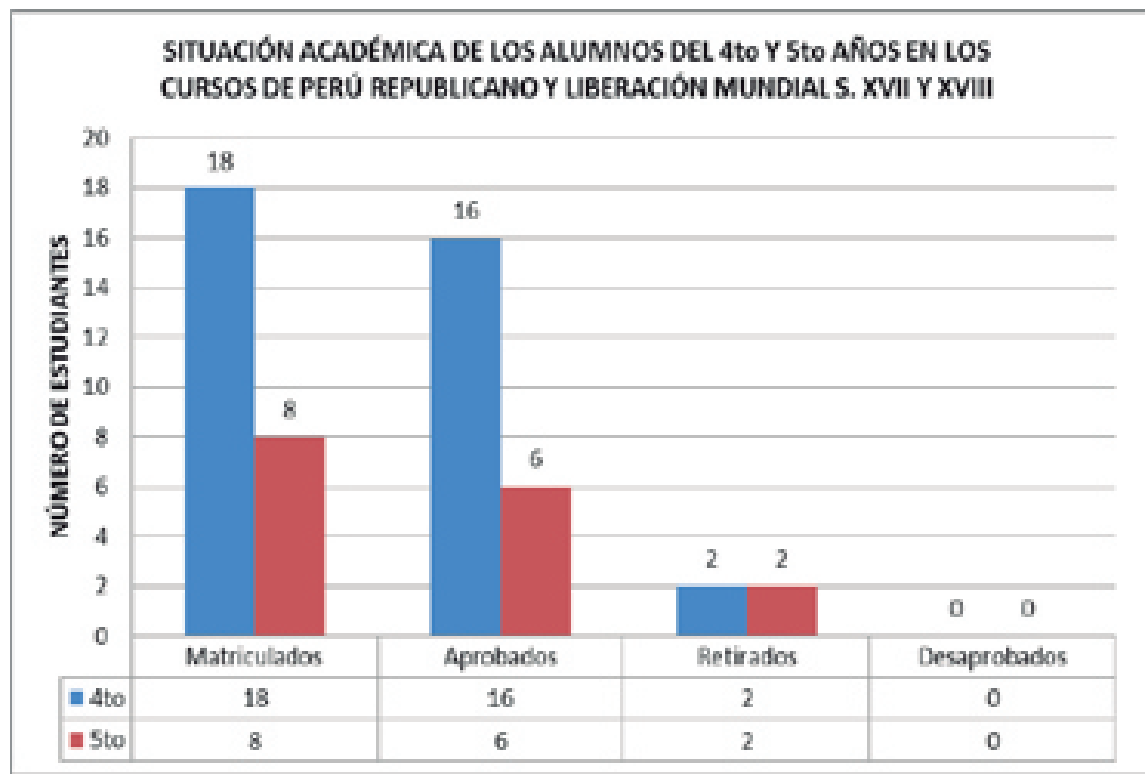

Se constata que, de los 18 alumnos matriculados en el 4to año, 16 lograron concluir sus estudios en el período regular, igualmente en el 5to año, se verifica que de los 8 estudiantes de la brigada, 6 concluyeron satisfactoriamente sus estudios. En ambos años de estudio se retiraron 2 estudiantes y ningunos fue desaprobado. Si bien es cierto que la población estudiantil es pequeña, la naturaleza de su permanencia en las aulas de clase es aceptable, considerando que frecuentemente la deserción en los últimos años es tiene un promedio de 40 a $50 \%$, conforme a la información estadística que maneja la Universidad. Las causas del abandono de su carrera universitaria, de acuerdo al historial académico de los alumnos, se deben a factores socioeconómicos en ambos grupos y también su disconformidad con la cátedra de los docentes. En los últimos cinco años en la Especialidad de Ciencias Histórico Sociales y Geográficas, la mayoría de los alumnos de los cursos de Perú Republicano y Liberación Mundial de los S. XVII y XVIII se observaba un buen número de desaprobados y repitentes, como un mal endémico. 
Tabla 2. Situación académica final de los alumnos en los cursos de Perú republicano y liberación mundial s. XVII y XVIII

\begin{tabular}{ccc}
\hline Frecuencias & 4 to & 5 to \\
\hline $14-18$ & 8 & 3 \\
$12-14$ & 6 & 2 \\
$11-12$ & 2 & 1 \\
\hline
\end{tabular}

FUENTE: Unidad de Procesos Académicos de la UNHEVAL, 2015

Figura 2. Situación académica final de los alumnos en los cursos de Perú republicano y liberación mundial s. XVII y XVIII

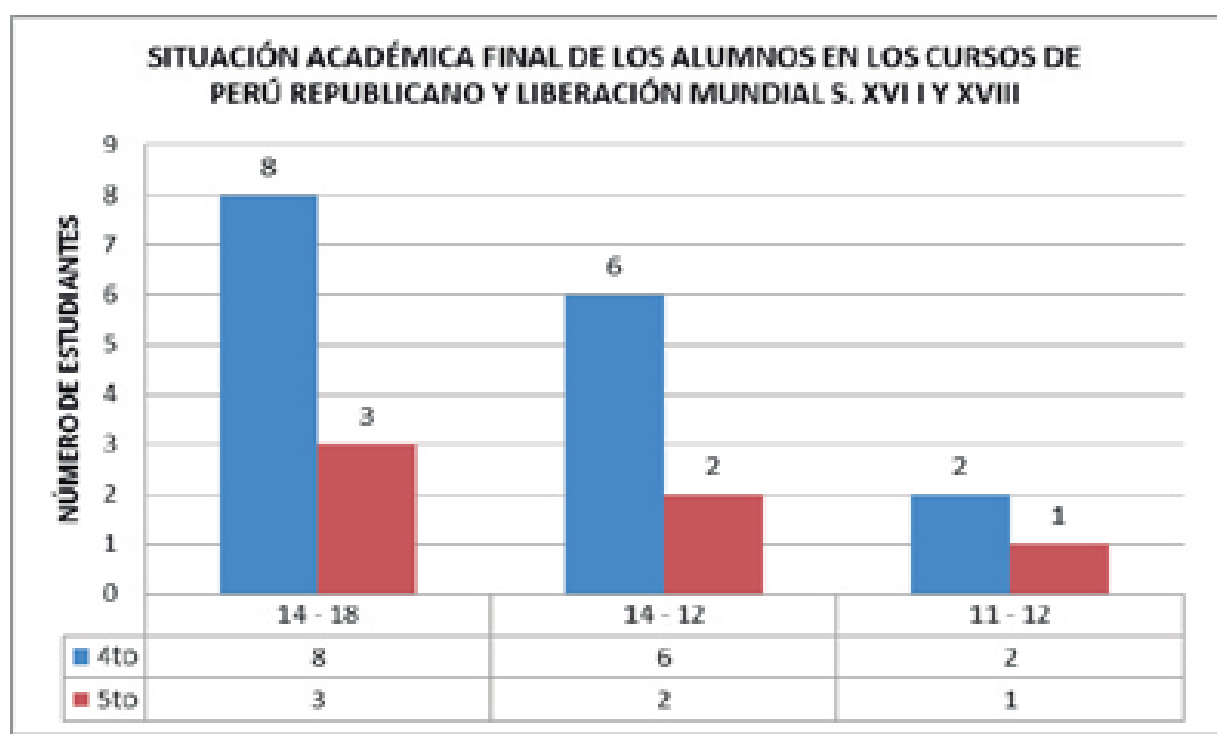

Igualmente en la tabla y la figura $\mathrm{N}^{\circ} .2$ podemos visualizar que el porcentaje de aprobados con notas de buena y muy buena son satisfactorios, considerando que sobrepasa el promedio histórico que era la nota 11 y 13, como se ha constatado en las actas promocionales de los últimos cinco años. Esto se debe esencialmente al cambio de estrategias metodológicas. Esta afirmación la constatamos en la entrevista que se hizo a los alumnos. Una de las estudiantes del cuarto año que, tiene bastante criticidad es Pedraza Quiñones (2015). Nos dice: "Los proyectos de aprendizaje me ha permitido adentrarme en el conocimiento de la historia en base a las fuentes de primera mano, por ejemplo en el Archivo Regional existe abundante información sobre Perú Republicano que yo desconocía sobre temas como la participación de Huánuco en la Guerra del Cenepa". Efectivamente, los alumnos visitaron el Archivo Regional con el fin de investigar temas referentes a Historia de Huánuco y realizaron trabajos monográficos con interesantes resultados, que hoy forman parte de la biblioteca de la Especialidad. Otro alumno, Anthony Quispe, con su agudeza de crítico, nos indicó que: "en los cinco años que estoy en la universidad si me hubieran exigido mis propios aprendizajes tal vez hubiera estado en otro nivel, lamentablemente recién en el 4to y 5 to años, usted está trabajando con 
los proyectos; si bien es cierto es trabajoso, pero nuestro aprendizaje es sustancioso". El alumno entrevistado dirige el Círculo Javier Pulgar Vidal, asociación de estudiantes dedicada a realizar trabajos de investigación Histórica y Geográfica. En un hecho poco usual en los estudiantes, presentó un trabajo monográfico sobre la historia de la emancipación con todas las formalidades del caso y con un contenido halagador. El exalumno Isaac Espinoza Padilla que culminó sus estudios en el 2014 en su despedida promocional al referirse a la Especialidad dijo: "Lo mejor que tuve en mi formación fueron los proyectos de aprendizaje y la elaboración de mis informes de investigación en el curso de Perú Republicano y mis primeras experiencias como docente con las prácticas preprofesionales con el profesor Raúl Aliaga Camarena, como parte del nuevo currículo. En ambos cursos tengo gratos recuerdos de aprendizaje, todos los cursos son buenos, pero lo que quedara para mi experiencia esos dos cursos". En la Facultad de Educación los alumnos realizan sus prácticas de internado en las comunidades, donde no solo realizan el dictado de clases, sino actividades de extensión y proyección social, trabajos de investigación en el Seminario de tesis; el hecho resaltante es el reconocimiento de sus aprendizajes a través de los proyectos en aula de clases. En otro momento entrevistamos a Pool Guzmán Laguna exalumno de la promoción 2013. Nos dice: "Profesor gracias por la entrevista, tengo muchos recuerdos de la universidad, pero lo que no me olvidaré nunca es haber escrito artículos, monografías e informes, con el rigor científico de las normas APA y Vancouver y sobre todo haber trabajado en el al Archivo Regional de Huánuco y haber visitado los monumentos arqueológicos de Kotosh, Kenaq, Garú para hacer mis trabajos; fue interesante y hoy lo hago lo mismo con mis alumnos". En verdad tenemos interesantes testimonios de los estudiantes y exalumnos, por el espacio que tenemos, no podemos citarlos a todos, cada uno de ellos han expresado sus pareceres en torno a la función que cumplen los proyectos de aprendizaje en el aula como parte de la gestión del conocimiento en los alumnos universitarios.

\section{DISCUSIÓN}

Nuestro resultado nos conduce a plantear argumentos teóricos, en el sentido de que el aprendizaje en la formación universitaria debe romper con los moldes y esquemas tradicionales que viene perdurando por años. Generar modelos de aprendizaje que articulen el uso de medios y herramientas personales, búsqueda de información, utilidad y satisfacción de aprendizajes, pues sabemos que existe variedad de información sobre los alcances de las nuevas estrategias de enseñanza-aprendizaje en las aulas universitarias. Sin embargo en nuestro país persiste la preponderancia del profesor que diserta sus clases en el aula; los libros de didáctica universitaria están orientados al desarrollo de contenidos temáticos y a las exposiciones magistrales. La Universidad Nacional Hermilio Valdizán no es ajena a estos moldes tradicionales en sus 28 carreras, muchas de sus escuelas profesionales han implementado el currículo basado en competencias, sin embargo en su aplicación siguen persistiendo con lo tradicional donde lo predominante es el contenido temático con la disertación expositiva de parte del docente. 
La especialidad de Ciencias Histórico Sociales y Geográficas como parte de sus planes de mejora, en diferentes eventos y certámenes ha discutido y viene discutiendo la problemática del desarrollo de competencias, aplicación de nuevas estrategias, el desempeño del futuro docente y sobre todo la gestión de nuevos conocimientos en el aula; por ello se plantea un aprendizaje basado en el uso de estrategias innovadoras en la gestión del conocimiento. Como es evidente los proyectos de aprendizaje son experiencias antiguas en las escuelas y colegios. Sin embargo en las aulas universitarias se ha notado muy poca presencia de estas propuestas, sobre todo en nuestro país.

Uno de los pocos que viene implementando los proyectos de aprendizaje con el enfoque Socioformativo es (Tobón, 2005) quien nos dice, la educación todavía sigue dando énfasis a la clase magistral la cual busca la transmisión de conocimientos desde una relación vertical, patriarcal y de sometimiento (p. 205). Compartimos esta afirmación, que válida nuestra propuesta descrito en el presente artículo. En otro pasaje de su teoría refiere:

La formación de instrumentos y estrategias afectivo motivacionales, cognitivometacognitivas y actuacionales requiere de transformaciones estructurales en las prácticas pedagógicas de las instituciones educativas, con el fin de formar auténticas comunidades de desarrollo de competencias donde todos aprendan de todos mediante la autorreflexión y la interacción social (Tobón, 2005, p.22).

Nuestra tesis y praxis de renovar las cátedras en las aulas por nuevas formas y estilos de aprendizaje concuerda, con los argumentos descritos por el autor de los proyectos formativos que, viene trabajando en México y Colombia. Sus afirmaciones se compatibilizan con los proyectos de aula en la gestión del conocimiento que venimos trabajando en nuestra Especialidad en la Universidad Valdizana en estos últimos dos años, donde los resultados son halagadores. Sin embargo, debemos indicar que nuestra experiencia en la gestión de los nuevos conocimientos está centrada en las aulas de clase de nivel universitario como las primeras experiencias, a diferencia del autor que nos plantea de manera general para todos los niveles académicos. Por su parte (Biggss, 2005) dice "La enseñanza "funciona" haciendo que los estudiantes se comprometan en actividades relacionadas con el aprendizaje y que les ayuden a alcanzar los objetivos concretos establecidos para la unidad o asignatura, como teorizar, crear nuevas ideas, reflexionar, aplicar, resolver problemas, memorizar, (pp.19-28). Compartimos su punto de vista, sin embargo el autor prioriza la reflexión académica dentro de las aulas de clase como una forma de aprendizaje, sobre ello diremos que sus argumentos son buenas, pero dependen de los contextos; en ese sentido, para Europa se encuadran a las necesidades estudiantiles, en tanto en América Latina, como el caso del Perú, no lo es así. Los estudiantes del tercer mundo optan por lo más fácil, lo que hay que hacer es que ellos adquieran compromisos con sus propios aprendizajes y en este caso los proyectos de aula son oportunidades excelentes para que desarrollen sus potencialidades y capacidades que les permitan lograr competencias y desempeños para el éxito de su vida profesional.

Un hecho sustantivo de nuestra experiencia es que el aprendizaje se da en diferentes niveles. En primera instancia está la metacognición del estudiante, es decir 
los saberes individuales. En base a esta experiencia los estudiantes se organizan en brigadas de aprendizaje, para luego elaborar su proyecto y ejecutarlo en la realidad ayudándose con el acopio de datos. El alumno para ello realiza una serie de actividades de manera personal y colectiva en los centros de información como: complejos arqueológicos, archivos, museos, bibliotecas, hemerotecas, navegar en páginas de la web, usa bibliotecas virtuales, visita comunidades, entrevista a las autoridades y personalidades, colecciona fotografías, elabora mapas, croquis, esquemas, intercambia experiencias; manejar herramientas como las Tic, Tac y el Tep, y hace uso de las redes sociales. Un segundo aspecto de su conocimiento es la socialización e intercambio de experiencias a nivel de su brigada, que concluye con la elaboración de sus informes, para luego pasar a la tercera fase del aprendizaje cuando las brigadas a través de sus representantes sustentan sus informes en plenaria de estudiantes, donde reciben críticas, felicitaciones, sugerencias; terminado esto pasan a la cuarta fase y en ella los estudiantes escriben artículos, monografías, tesinas, informes y finalmente desarrollan la quinta fase del aprendizaje, espacio en que, los trabajos son sometidos a una evaluación por parte del docente con presencia de los miembros de las brigadas, quienes reciben sugerencias y observaciones si el caso amerita. A estos procedimientos lo denominamos gestión del conocimiento en el aula. Un hecho singular de este proceso es que los estudiantes ya no repiten lo que está escrito en los repositorios de la información como libros o textos universitarios, sino que ellos mismos van adquiriendo nuevos conocimientos como parte del desarrollo de su proyecto de aula, en la que muchas veces sus resultados son diferentes a los que convencionalmente se afirmaba.

Otro rasgo destacable es el uso y la práctica de nuevas estrategias de aprendizaje, como el trabajo de las brigadas, que, Tobón considera como el aprendizaje colaborativo, tesis con la que concordamos en muchos aspectos, porque tanto el aprendizaje colaborativo y aprendizajes a través de las brigadas estudiantiles tienen similitudes en las formas de recoger la información, pero con la diferencia que los aprendizaje en las brigadas a través de proyectos de aula cumplen varios niveles para consolidar los nuevos saberes como se ha descrito en la cartografía.

En los proyectos de aula, los estudiantes adquieren una cultura investigativa, desde los procesos más elementales hasta consolidar sus trabajos en artículos de nivel científico. Nuestra experiencia nos dice que los estudiantes en el campo de la historia logran desarrollar capacidades como juicio crítico, identifica y utiliza apropiadamente fuentes de información bibliográfica, almacenamiento documental en el sistema virtual, testimonios orales para la investigación histórica; toma conciencia de que el debate y la investigación histórica están en permanente construcción. En el campo geográfico explica las relaciones entre los elementos naturales y culturales que participan en la construcción de los espacios geográficos, evalúa problemáticas ambientales y territoriales desde múltiples perspectivas, precisa situaciones de riesgo y propone acciones para disminuir la vulnerabilidad frente a los desastres, maneja y elabora diversas fuentes de información y herramientas digitales para comprender el espacio geográfico; asimismo, en el componente economía, comprende las relaciones entre los elementos del sistema económico y financiero, 
toma conciencia de que es parte de un sistema. Si bien es cierto el desarrollo de las capacidades referidas es lento, pero son de mucha utilidad para los estudiantes, toda vez que sus aprendizajes están orientados al logro de competencias para una, buen desempeño profesional que la región de Huánuco y el país lo necesitan. Un hecho particular que nos ha permitido observar de cerca es la innovación de estrategias por parte de los brigadistas, en relación al recojo de información; mencionamos algunas: las discusiones alturadas de sus aportes, el uso de celulares como instrumentos para el recojo de información y las redes sociales para el intercambio de opiniones y datos. Benites ( 2010) nos dice que las Tecnologías de la Información y la Comunicación (TIC) jueguen un papel fundamental en este nuevo espacio, como herramientas que pueden facilitar tanto la implementación de nuevas metodologías como el aprendizaje del alumno y la comunicación interpersonal alumno-alumno y alumno-profesor (p. 2). Efectivamente es un interesante recurso; a los docentes nos permite monitorear el trabajo a través de las redes sociales como el Twitter, el Facebook, el chat, el foro y sobre todo el Skype que nos posibilita interactuar dentro y fuera del aula, asesorando y orientando a los estudiantes en sus dificultades académicas de formación profesional. El planteamiento de Benites concuerda con nuestra experiencia toda vez que los futuros profesionales vienen logrando el manejo de las competencias tecnológicas, pedagógicas, comunicativas, de gestión y de investigación, que son los pilares básicos de todo docente de nuestros tiempos; de allí la importancia de esta estraegia que viene trabajando la especialidad de Ciencias Histórico Sociales y Geográficas de manera experimental.

\section{CONCLUSIONES}

1) El proyecto de aula en clase es un estrategia de primer orden que permite a los brigadistas gestionar sus aprendizajes y consiguientemente los nuevos conocimientos.

2) Las brigadas estudiantiles es una alternativa didáctica que los docentes de las aulas universitarias debemos utilizar para dinamizar el aprendizaje de los estudiantes, toda vez que permite detectar el talento humano, la creatividad, innovación y el emprendimiento.

3) El proyecto de aula en clase, como alternativa didáctica sirve para discernir la información, pues en su proceso pasa por cinco niveles: trabajo individual, aprendizajes colaborativos, sustentación y argumentación en plenaria, la producción de conocimientos (redacción y producción de textos), socialización del conocimiento que se manifiesta en la publicación del conocimiento.

4) Los proyectos de aula posibilitan a los brigadistas el uso y manejo de las tecnologías de información y comunicación (TIC), así como las tecnologías del aprendizaje y conocimiento (TAC) y las Tecnologías de las estrategias de producción (TEP).

5) Igualmente los brigadistas logran desarrollar competencias para el adecuado desempeño profesional, (convivencia, asertividad, actitud propositiva) que le exige el mercado ocupacional del tercer milenio. 
6) Los estudiantes como producto y manejo de los proyectos de aula en clase adquieren competencias investigativas.

\section{REFERENCIAS BIBLIOGRÁFICAS}

Benítez, M. (2010). Aprendizaje basado en problemas a través de las TIC. Málaga: Universidad de Málaga.

Biggs, J (2005). Calidad del aprendizaje universitario. Madrid: Narcea S. A.

Diez Canseco, J. (1984). Actitudes docentes y formación del magisterio. Recuperado de https://dialnet.unirioja.es/descarga/articulo/263582.pdf..

Marquesán, C. (2008). Valores en el contexto de la educación para la ciudadanía. En Formación de docentes en educación de valores y ciudadanía. Colombia: Instituto para el Desarrollo y la Innovación Educativa, IDIE - Formación de docentes y educadores.

Ministerio de Educación.(2015). Diseño curricular nacional. Lima: Ministerio de Educación.

Restrepo, B.(s.f). Conceptos y aplicaciones de la investigación formativa, y criterios para evaluar la investigación científica en sentido estricto. Colombia. Recuperado de http://www.cna.gov.co/1741/articles-186502_doc academico. pdf.

Tobón, S. (2013). Los proyectos formativos: transversalidad y desarrollo de competencias para la sociedad del conocimiento. México: Instituto CIFE.

Tobón. S. (2005). Formación basada en competencias: Pensamiento complejo, diseño curricular y didáctica. Colombia. Ecoe Ediciones.

Universidad Politécnica de Madrid. (2008). Aprendizaje orientado a proyectos. Guías rápidas sobre nuevas metodologías. Madrid: Servicio de innovación educativa de la Universidad Politécnica de Madrid.

UNHEVAL (2015). Informe de la Comisión de Currículo y Homologación. Facultad de Ciencias de la Educación. Huánuco.

UNHEVAL (2014). Reglamento del estudiante. Universidad Nacional Hermilio Valdizán.

UNHEVAL (2014). Informe Curricular de la Facultad de Ciencias de la Educación. Universidad Nacional Hermilio Valdizán. Huánuco.

Zabalza, M. (2005). Conferencia pronunciada en la Pontificia Universidad Javeriana de Cali. 